\title{
A Modified Right Helicoid Can Simulate the Inner Structure of the Cochlea in the Hearing Organ of Mammals
}

\author{
Motohisa Osaka \\ Department of Basic Science, Nippon Veterinary and Life Science University, Tokyo, Japan \\ Email: osaka@nms.ac.jp
}

How to cite this paper: Osaka, M. (2021) A Modified Right Helicoid Can Simulate the Inner Structure of the Cochlea in the Hearing Organ of Mammals. Applied Mathematics, 12, 399-406. https://doi.org/10.4236/am.2021.125028

Received: April 16, 2021

Accepted: May 16, 2021

Published: May 19, 2021

Copyright $\odot 2021$ by author(s) and Scientific Research Publishing Inc. This work is licensed under the Creative Commons Attribution International License (CC BY 4.0).

http://creativecommons.org/licenses/by/4.0/

\section{(c) (i) Open Access}

\begin{abstract}
The purpose of this study is to develop a mathematical model of the spiral basilar membrane in the center of the cochlea, which plays an important role in the mammalian auditory system. The basilar membrane transmits sound vibrations, which are converted into electrical potential changes by the inner hair cells. The basilar membrane is thought to lie on a locally undistorted curved surface because the inner hair cells, which are arranged in an orderly fashion on the basilar membrane, respond to their location-specific frequencies. In mammals, the number of rotations of this surface and the rate of change of its width with each rotation are different. It turns out that by modifying the right helicoid, we can obtain a mathematical model that satisfies these points. In conclusion, even though the three-dimensional structure of the basilar membrane varies among species, this model can reproduce this structure. This further suggests that there are common genetic determinants of cochlear development in mammals. From a practical standpoint, this may be useful for creating cochlear implants.
\end{abstract}

\section{Keywords}

Auditory Organ, Cochlea, Right Conoid, Mean Curvature

\section{Introduction}

It may come as a surprise to learn that mammals have a sensory organ in their auditory system that is similar in shape to a snail. During the course of evolution, mammals acquired a snail-shaped cochlea (cochlea is Greek for snail) in their hearing organ [1] [2]. Therefore, it has been speculated that they have a superior auditory structure. In order to reproduce in a mathematical model the 
structure of the basilar membrane of the inner ear, which is particularly important as an auditory organ, the structure of the inner ear is first described in detail below.

The ear is divided into three main parts: the outer ear, the middle ear, and the inner ear. The outer ear consists of the auricle and ear canal, with the eardrum at the far end of the ear canal. Behind the eardrum is the middle ear, which consists of a small chamber called the tympanic chamber surrounded by bone. Here are three small bones that form a bridge between the eardrum and the inner ear. The inner ear is buried deep within the bone and consists of the cochlea, which is responsible for hearing, and the vestibule, which is responsible for balance.

The cochlea is a spiral canal structure that makes about 2.75 turns around the cochlear axis and is approximately $35 \mathrm{~mm}$ long in humans when stretched is extended and about $2 \mathrm{~mm}$ thick at the base of the middle ear. The interior of the cochlea consists of three compartments separated by membranes along the direction of the whirlpool: the vestibular level, the central level, and the tympanic level. The vestibular and tympanic floors are connected by the apex at the end of the cochlear duct and are both filled with perilymph, whereas the central floor is filled with endolymph, which is rich in potassium ions due to active transport of ions. The basilar membrane has a natural frequency that corresponds to lower tones as it moves from the base to the apex. At the central level of the basilar membrane is an orderly array of small, delicate and sturdy structures called the organ of Corti. In the upper part of the organ of Corti, two types of hair cells, called inner and outer hair cells, are regularly arranged along the cochlear canal. In humans, there are about 3500 inner hair cells and 15,000 to 20,000 outer hair cells in one ear. The hair cells are arranged in four rows in the organ of Corti along the entire length of the cochlea. Three rows consist of outer hair cells and one row consists of inner hair cells. The inner hair cells provide the main neural output of the cochlea. The inner hair cells are the primary sensory receptors that convert vibration information into nerve pulses. The central axis of the cochlea contains numerous cochlear ganglia, which form synaptic connections with the inner hair cells. The axons of these neurons form the cochlear nerve, which projects to several cochlear nuclei that span the medulla oblongata and the pons in the brain. Instead, the outer hair cells mainly receive neural input from the brain, which influences their motility as part of the cochlea's mechanical preamplifier.

Based on the above, the structure of the cochlear canal can be summarized as follows: 1) the cochlear canal rotates in a spiral from the bottom to the apex around the cochlear axis in mammals, and it makes about 3 turns in humans, 2) sound-induced air vibrations are transmitted from the middle ear to the inner hair cells, which are arranged in an orderly fashion on the basilar membrane of the cochlear duct, via lymphatic fluid, and are converted into electrical signals that are transmitted to nerves in the central axis of the cochlear duct, and 3) the outer hair cells mainly receive neural input from the brain. 
The above features of the cochlea are common to all mammals. Since the central axis of the cochlea has afferent nerves leading to the inner hair cells, and the outer hair cells have centrifugal nerves from the brain, the purpose of this study is to propose a mathematical model that reproduces the rotation of the basilar membrane around the cochlear axis. If structural variations of the basilar membrane in different mammalian species can be reproduced by simply changing the parameters of this model, it suggests the possibility that there are rules common to mammals in the development of the cochlea. In other words, it suggests that common gene loci that generate the cochlea are present in mammals. This mathematical model may help determine the structure of the cochlea when it is artificially regenerated in the future.

\section{Mathematical Model and Simulation}

The purpose of this study is to develop a mathematical model that meets the following requirements in accordance with the actual structure of the basilar membrane.

1) A spiral surface reproduces how the basilar membrane rotates around the central axis from the bottom to the apex.

2) Since the hair cells are arranged in an orderly fashion on the basilar membrane, the spiral surface has no local distortion.

3) Because the external shape of the cochlea is like that of a snail, with a broad base and narrowing toward the apex, the surface on which the basilar membrane rests gradually narrows toward the apex.

4) The number of rotations can vary [2].

5) The rate of change of the basilar membrane width with each rotation can vary (the width of the spiral surface is narrower in humans than in guinea pigs as it rotates [2]).

A surface rotating spirally around a central axis can be represented by a ruled surface, which is a surface created by a family of straight lines depending on one parameter. The structure of this ruled surface meets the first requirement. Suppose that the central axis of the spiral surface is on the z-axis in a three-dimensional space $x-y-z$. The nerve is connected perpendicularly from the central axis to the spiraling basilar membrane of the actual cochlea. Therefore, we can assume that this ruled surface is formed from a family of straight lines intersecting perpendicularly to the z-axis. These are expressed in a mathematical form called as right conoid:

$$
x=u \cos v, y=u \sin v, z=f(v) .
$$

Then,

$$
\begin{gathered}
\boldsymbol{p}(u, v)=(u \cos v, u \sin v, f(v)), \\
\boldsymbol{p}_{u}=(\cos v, \sin v, 0), \boldsymbol{p}_{v}=\left(-u \sin v, u \cos v, f^{\prime}(v)\right), \\
\boldsymbol{p}_{u u}=(0,0,0), \boldsymbol{p}_{u v}=\boldsymbol{p}_{v u}=(-\sin v, \cos v, 0), \boldsymbol{p}_{v v}=\left(-u \cos v,-u \sin v, f^{\prime \prime}(v)\right)
\end{gathered}
$$




$$
\boldsymbol{e}=\frac{\boldsymbol{p}_{u} \times \boldsymbol{p}_{v}}{\left|\boldsymbol{p}_{u} \times \boldsymbol{p}_{v}\right|}=\frac{1}{\Delta}\left(f^{\prime}(v) \sin v,-f^{\prime}(v) \cos v, u\right) ; \Delta=\sqrt{u^{2}+f^{\prime}(v)^{2}} .
$$

For the first and second fundamental forms of $p(u, v)$,

$$
\begin{aligned}
& I=\mathrm{d} \boldsymbol{p} \cdot \mathrm{d} \boldsymbol{p}=E \mathrm{~d} u \mathrm{~d} u+2 F \mathrm{~d} u \mathrm{~d} v+G \mathrm{~d} v \mathrm{~d} v, \\
& I I=-\mathrm{d} \boldsymbol{p} \cdot \mathrm{d} \boldsymbol{e}=L \mathrm{~d} u \mathrm{~d} u+2 M \mathrm{~d} u \mathrm{~d} v+N \mathrm{~d} v \mathrm{~d} v .
\end{aligned}
$$

Therefore,

$$
\begin{gathered}
E=1, F=0, G=u^{2}+f^{\prime}(v)^{2}, \\
L=0, M=-\frac{f^{\prime}(v)}{\Delta}, N=\frac{u f^{\prime \prime}(v)}{\Delta} .
\end{gathered}
$$

For the principal curvatures, $\kappa_{1}$ and $\kappa_{2}$, at the given point, the Gaussian curvature $K$ and the mean curvature $H$ of this surface are expressed as follows.

$$
\begin{gathered}
K=\kappa_{1} \kappa_{2}=\frac{L N-M^{2}}{E G-F^{2}}, \\
H=\frac{1}{2}\left(\kappa_{1}+\kappa_{2}\right)=\frac{E N+G L-2 F M}{2\left(E G-F^{2}\right)} . \\
K=-\frac{f^{\prime}(v)^{2}}{\left(u^{2}+f^{\prime}(v)^{2}\right)^{2}}, H=\frac{u f^{\prime \prime}(v)}{2\left(u^{2}+f^{\prime}(v)^{2}\right)^{3 / 2}} .
\end{gathered}
$$

A surface is said to be minimal if its area becomes larger than the original surface when it is slightly deformed in the direction of the normal vector at an arbitrary point. The necessary and sufficient condition for this to be the case is $H$ $=0$. If $H=0$, then the mean curvature is zero at that point, and in that sense, a minimal surface is a surface without local distortion. So, according to the second requirement, the condition for $H=0$ is as follows.

$$
H=0 \Leftrightarrow f^{\prime \prime}(v)=0 \Leftrightarrow f(v)=a v+b ; a \text { and } b \text { are constants. }
$$

When $f(v)=a v+b$, this surface is called a right helicoid. We illustrate the right helicoid when $f(v)=v$ (Figure 1). In Figure 1, the surface rises in an evenly spiraling rotation around the central axis.

The mathematical model of the right helicoid is modified as follows to match this shape to the third requirement.

$$
\begin{gathered}
x=u g(v) \cos v, \\
y=u g(v) \sin v, \\
z=f(v), \\
g(v) \equiv-c f(v)+1 ;
\end{gathered}
$$

$c$, positive constant.

Figure 2 is a modified version of Figure 1, showing a so-called snail-shaped curved surface that tapers from the wide bottom to the apex.

Then, 


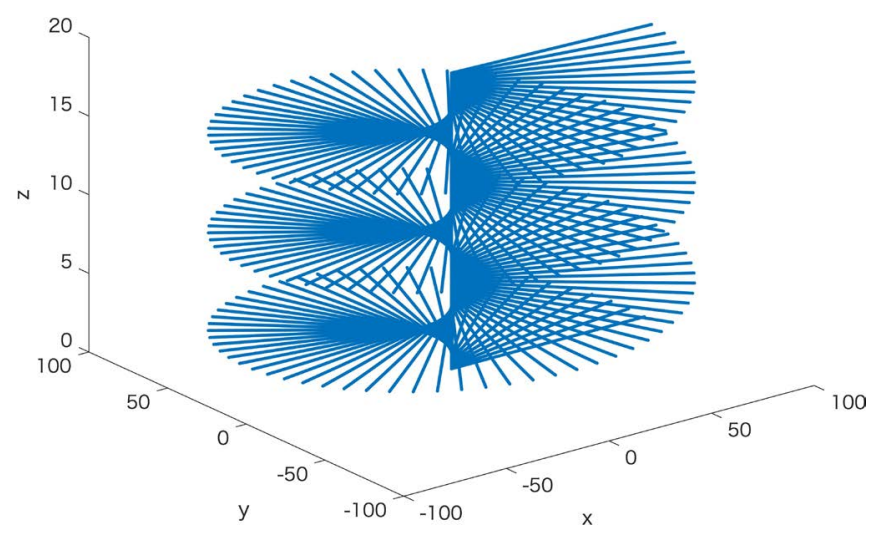

Figure 1. Example of a right helicoid; $f(v)=v, 0 \leq u \leq 94.5,0 \leq v \leq 6 \pi$. The curved surface rises in an even spiral rotation around the central axis.

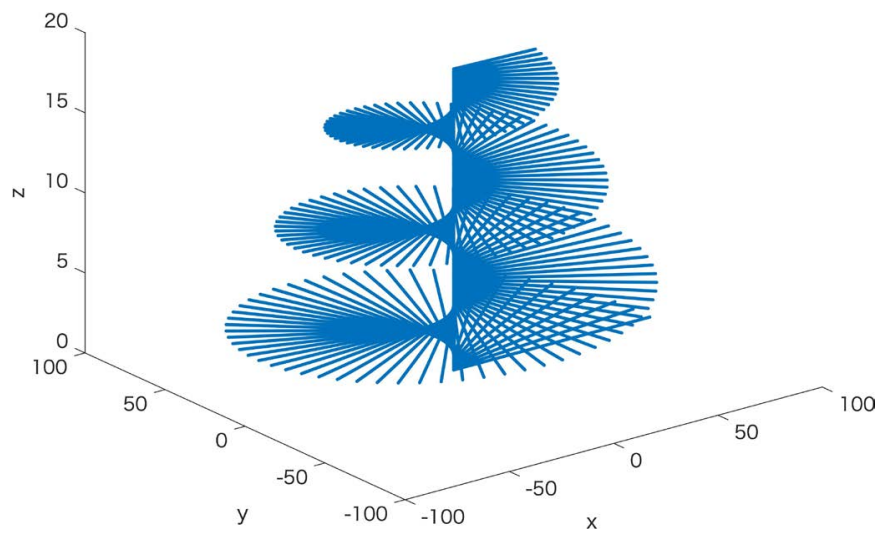

Figure 2. A modified version of the right helicoid of Figure 1. $f(v)=v, 0 \leq u \leq 94.5$, $0 \leq v \leq 6 \pi, c=1 / 10 \pi$.

$$
\begin{gathered}
\boldsymbol{p}(u, v)=(u g(v) \cos v, u g(v) \sin v, f(v)), \\
\boldsymbol{p}_{u}=(g(v) \cos v, g(v) \sin v, 0), \\
\boldsymbol{p}_{v}=\left(-u g(v) \sin v-c u f^{\prime}(v) \cos v, u g(v) \cos v-c u f^{\prime}(v) \sin v, f^{\prime}(v)\right), \\
\boldsymbol{p}_{u u}=(0,0,0), \\
\boldsymbol{p}_{u v}=\boldsymbol{p}_{v u}=\left(-g(v) \sin v-c f^{\prime}(v) \cos v,\right. \\
\boldsymbol{p}_{v v}=\left(-u g(v) \cos v-c f^{\prime}(v) \sin v, 0\right), \\
\left.-u g(v) \sin v-2 c u f^{\prime}(v) \cos v-c u f^{\prime \prime}(v) \sin v, f^{\prime \prime}(v)\right) . \\
\boldsymbol{e}^{-}=\frac{\boldsymbol{p}_{u} \times \boldsymbol{p}_{v}}{\left|\boldsymbol{p}_{u} \times \boldsymbol{p}_{v}\right|}=\frac{1}{\Delta}\left(f^{\prime}(v) \sin v,-f^{\prime}(v) \cos v, u g(v)\right) \\
\Delta=\sqrt{u^{2} g(v)^{2}+f^{\prime}(v)^{2}} \cdot \\
E=g(v)^{2}, F=-c u g(v) f^{\prime}(v),
\end{gathered}
$$




$$
\begin{gathered}
G=u^{2} g(v)^{2}+\left(c^{2} u^{2}+1\right) f^{\prime}(v)^{2} \\
L=0, M=-\frac{g(v)^{2} f^{\prime}(v)}{\Delta}, \\
N=\frac{u\left[2 c f^{\prime}(v)^{2}+f^{\prime \prime}(v) g(v)\right]}{\Delta}
\end{gathered}
$$

Then,

$$
\begin{gathered}
K=-\frac{f^{\prime}(v)^{2}}{\left[u^{2} g(v)^{2}+f^{\prime}(v)^{2}\right]^{2} g(v)^{2}}, \\
H=\frac{u f^{\prime \prime}(v) g(v)}{2\left[u^{2} g(v)^{2}+f^{\prime}(v)^{2}\right]^{3 / 2}}
\end{gathered}
$$

In this modified model, as long as $f^{\prime \prime}(v)=0, H=0$ and the second requirement is satisfied. Then, $f(v)=a v+b$. Moreover, the shape of the spiral surface is snail-shaped, which satisfies the third requirement. As the range in which $v$ varies is increased, the number of rotations increases proportionally. Hence, the fourth requirement is possible with this model. For example, this model can reproduce a spiral surface with two rotations (Figure 3). Since $f(v)=v$ does not lose its generality, we will assume $f(v)=v$ for simplicity. Then, $g(v)=1$ when $v=0$, and as $v$ increases, $g(v) \equiv-c v+1$ decreases correspondingly from 1 . Hence, as $V$ increases, the spiral surface rises, so if $c$ is large, the width of the spiral surface narrows correspondingly. On the other hand, if $c$ is small, it rotates upward while maintaining a relative width. Since the value of $c$ in Figure 4 is smaller than the value of $c$ in Figure 3, the width of the spiral surface does not become so narrow even though it rises while rotating. In Figure 4, the number of turns is set to 4 , so the spiral surface reproduces the shape of the cochlea of guinea pig.

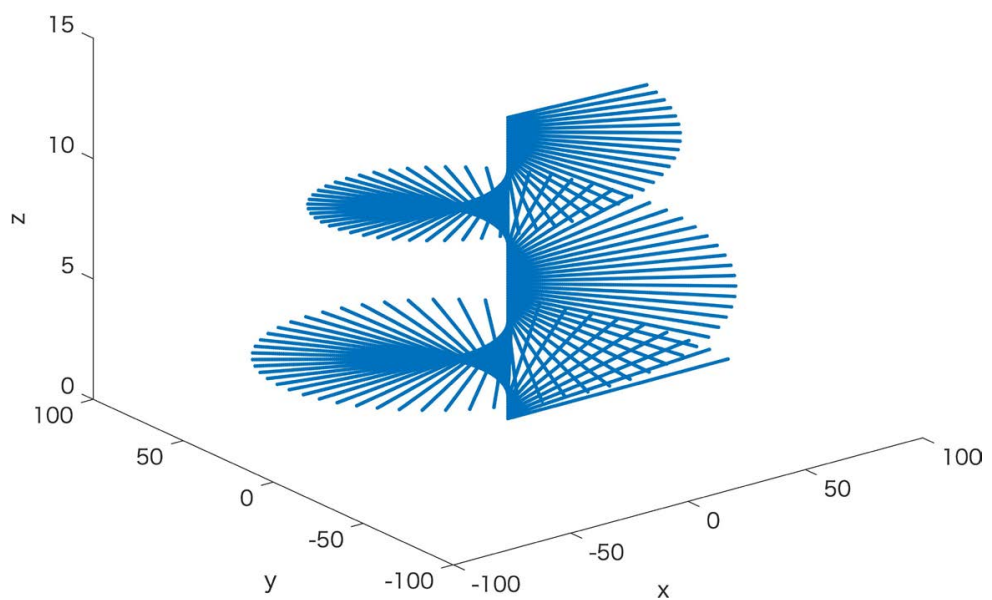

Figure 3. A modified version of a right helicoid of Figure 1. $f(v)=v, 0 \leq u \leq 94.5$, $0 \leq \nu \leq 4 \pi, c=1 / 10 \pi$. 


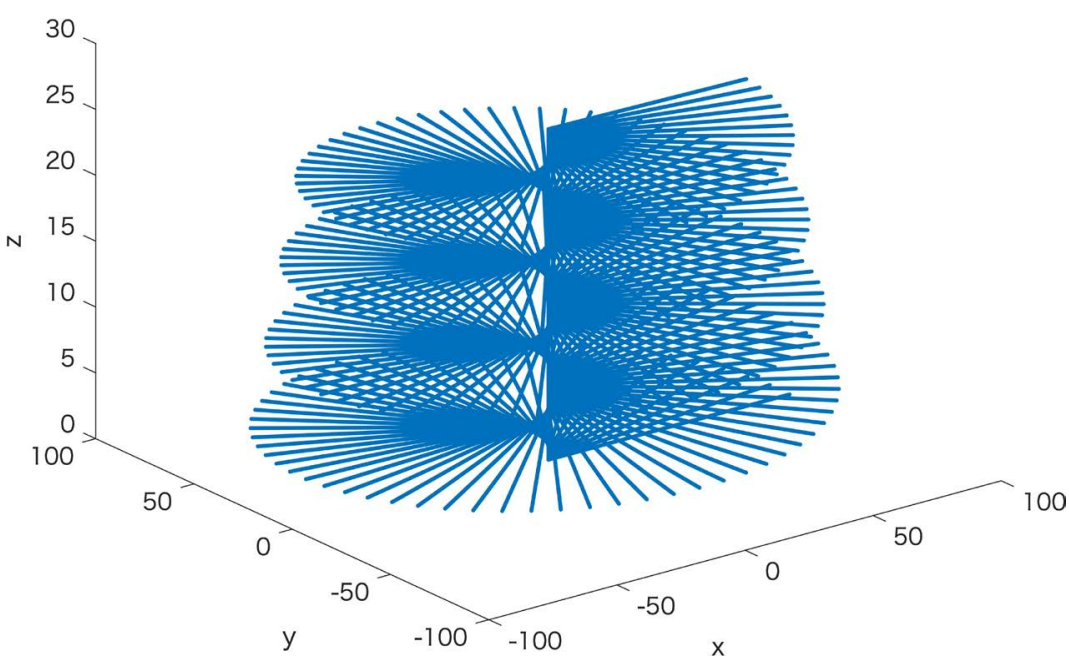

Figure 4. A modified version of a right helicoid of Figure 1. $f(v)=v, 0 \leq u \leq 94.5$, $0 \leq v \leq 8 \pi, c=1 / 40 \pi$.

\section{Discussion}

The Raup model is a revolutionary mathematical model of the morphology of snails published in 1966 [3]. Our model does not reproduce the external shape of the cochlea, which has the shape of a snail. The purpose of our model is to reproduce the spiral surface on which the inner hair cells, which are the most important part of the auditory system, are arranged.

The basic structure of the mammalian cochlea is the same. The inner hair cells, which are arranged on the basilar membrane, convert their location-specific frequencies into electrical signals. In order for this to be done accurately, the inner hair cells are arranged in an orderly fashion on the basilar membrane. We have found that our modified model can reproduce a locally undistorted surface at any point. Moreover, although there are differences in the number of basilar membrane rotations and the rate of change of the basilar membrane width with each rotation among mammalian species [2], the former can be reproduced by changing the parameter $v$ and the latter by changing the parameter $c$. The number of rotations can be adjusted by the range of $v$. The term $g(v) \equiv-c f(v)+1$ was used to deform the outer shape of the right helicoid into a snail shape. By increasing or decreasing the value of $c$, the external shape of the snail can be changed from an elongated snail-like shape to a blunt shape. The mean curvature was still able to maintain zero with $g(v)$, guaranteeing that there is no local distortion.

The fact that our model was able to reproduce the surface on which the mammalian inner hair cells are mounted suggests that there are common rules for the development of the mammalian cochlea. Considering that the gene loci of the cochlea have been identified from experiments with cochlear phenotypes of mutant and knockout mice [4] [5], our model suggests that these gene loci are probably common to all mammals. 


\section{Conclusion}

Our model, which was modified from the right helicoid, reproduced the spiral surface on which the basilar membrane is placed without local distortion. In addition, this model has the advantage of being able to reproduce the variation in the number of rotations and the width of the spiral surface among species. These findings suggest that there are common genetic determinants of cochlear development in mammals. The mathematical model may be useful in the creation of cochlear implants.

\section{Conflicts of Interest}

The author declares no conflicts of interest regarding the publication of this paper.

\section{References}

[1] Romer, A.S. (1957) Man and the Vertebrates. University of Chicago Press, Chicago.

[2] Ekdale, E.G. (2013) Comparative Anatomy of the Bony Labyrinth (Inner Ear) of Placental Mammals. PLOS ONE, 10, e0137149.

https://doi.org/10.1371/journal.pone.0066624

[3] Raup, D.M. (1966) Geometric Analysis of Shell Coiling: General Problems. Journal of Paleontology, 40, 1178-1190.

[4] Cantos, R., Cole, L.K., Acampora, D., Simeone, A. and Wu, D.K. (2000) Patterning of the Mammalian Cochlea. Proceedings of the National Academy of Sciences of the United States of America, 97, 11707-11713. https://doi.org/10.1073/pnas.97.22.11707

[5] Choo, D., Ward, J., Reece, A., Dou, H., Lin, Z. and Greinwald, J. (2006) Molecular Mechanisms Underlying Inner Ear Patterning Defects in Kreisler Mutants. Developmental Biology, 289, 308-317. https://doi.org/10.1016/j.ydbio.2005.10.007 